\title{
Epigenetic Alterations and Cancer
}

\author{
Mojgan Naghitorabi ${ }^{1,}$ \\ ${ }^{1}$ Department of Pharmacognosy, School of Pharmacy, Ahvaz Jundishapur University of Medical Sciences, Ahvaz, IR Iran \\ "Corresponding author: Mojgan Naghitorabi, Department of Pharmacognosy, School of Pharmacy, Ahvaz Jundishapur University of Medical Sciences, Ahvaz, IR Iran. Tel: \\ +98-9163003139, Fax: +98-6133738381, E-mail: mnaghitorabi@gmail.com \\ Received 2018 April 21; Accepted 2018 April 28.
}

Keywords: DNA Methylation, Histone Modification, miRNA, Cancer, Epigenetic

Epigenetic alterations are considered as any changes in the gene function that are heritable and do not involve a change in nucleotide sequence of DNA. Epigenetic changes participate in crucial biological processes such as X-chromosome inactivation, genomic imprinting, cell reprogramming during differentiation, and regulation of gene expression in eukaryotic organisms. In contrast to genetic changes that are very stable and strict, epigenetic changes can be reversible and highly dynamic, playing a major role in cellular regulating processes. In fact various epigenetic mechanisms control the accessibility of DNA to transcription factors and enzymes by affecting the chromatin conformation. Three main mechanisms for epigenetic regulation of gene expression are DNA methylation, histone modifications, and RNA mediated gene silencing $(1,2)$.

DNA methylation is the most common epigenetic change that plays a critical role in regulating gene expression. This modification is brought about by the addition of a methyl onto cytosine within CG dinucleotides called CPG in DNA and is catalyzed by DNA methyltransferases (DNMTs). In mammalian cells, $60 \%-70 \%$ of CpGs are methylated. Unmethylated CpGs are located in the 5 ' regulatory regions (promoters and the first exons) of many genes, as clusters called $\mathrm{CpG}$ islands. The promoter regions in transcriptionally active genes are normally unmethylated. It has been shown that DNA methylation pattern in tumor tissues differs largely relative to their corresponding normal tissues. Cancer cells commonly represent global hypomethylation, along with regional hypermethylation at the promoter regions of tumor suppressor, and DNA repair genes $(1,3,4)$.

Histone modifications have recently become the focus of interest in cancer research. The $\mathrm{N}$ terminal extensions of histone proteins may undergo several different modifications such as acetylation, methylation, and phospho- rylation. The coexistence of multiple and diverse histone modifications at the promoter regions of genes provide an elaborate dynamic epigenetic control of gene expression. It has been supposed that, histone modifications collaborate with DNA methylation during gene silencing $(1,2)$.

The third epigenetic mechanism is RNA mediated gene silencing, a natural mechanism that is triggered by the formation of small double stranded RNAs called microRNAs (miRNAs) in the cell. These miRNAs can guide a specific silencing complex either to their complementary mRNAs to mediate the translation inhibition or mRNA degradation,or to specific DNA sequences at the promoter region of genes, where they can lead to facilitating histone modifications and DNA methylation and subsequent gene silencing. Due to the pivotal roles of miRNAs in cellular biological processes, their dysregulation can lead to diseases including cancer $(1,5)$.

Nowadays many attempts are being performed on understanding the epigenetic basis of carcinogenesis, investigating epigenetic biomarkers as a diagnostic or prognostic tool in cancer, and even targeting the epigenetic defects as a potential cancer therapy.

\section{References}

1. Nap JP, Kessel AG. Epigenetics in context. Plant Research International. Wageningen, Netherlands; 2006. p. 15-45.

2. Bhattacharjee D, Shenoy S, Bairy KL. DNA Methylation and Chromatin Remodeling: The Blueprint of Cancer Epigenetics. Scientifica (Cairo). 2016;2016:6072357. doi: 10.1155/2016/6072357. [PubMed: 27119045]. [PubMed Central: PMC4826949].

3. Szyf M. Targeting DNA methylation in cancer. Bull Cancer. 2006;93(9):961-72. [PubMed: 16980240].

4. Ehrlich M. DNA methylation in cancer: too much, but also too little. Oncogene. 2002;21(35):5400-13. doi: 10.1038/sj.onc.1205651. [PubMed: 12154403].

5. Suzuki H, Maruyama R, Yamamoto E, Kai M. Epigenetic alteration and microRNA dysregulation in cancer. Front Genet. 2013;4:258. doi: 10.3389/fgene.2013.00258. [PubMed: 24348513]. [PubMed Central: PMC3847369]. 